\title{
Risk factor analysis for in-hospital death of geriatric hip fracture patients
}

Ping Zhang, MD, Xinping Li, MD, Yuan Yuan, MD, Xiaoyu Li, MD, Xiaoyan Liu, MD, Bin Fan, MD, Minghui Yang, MD, Xinbao Wu MD.

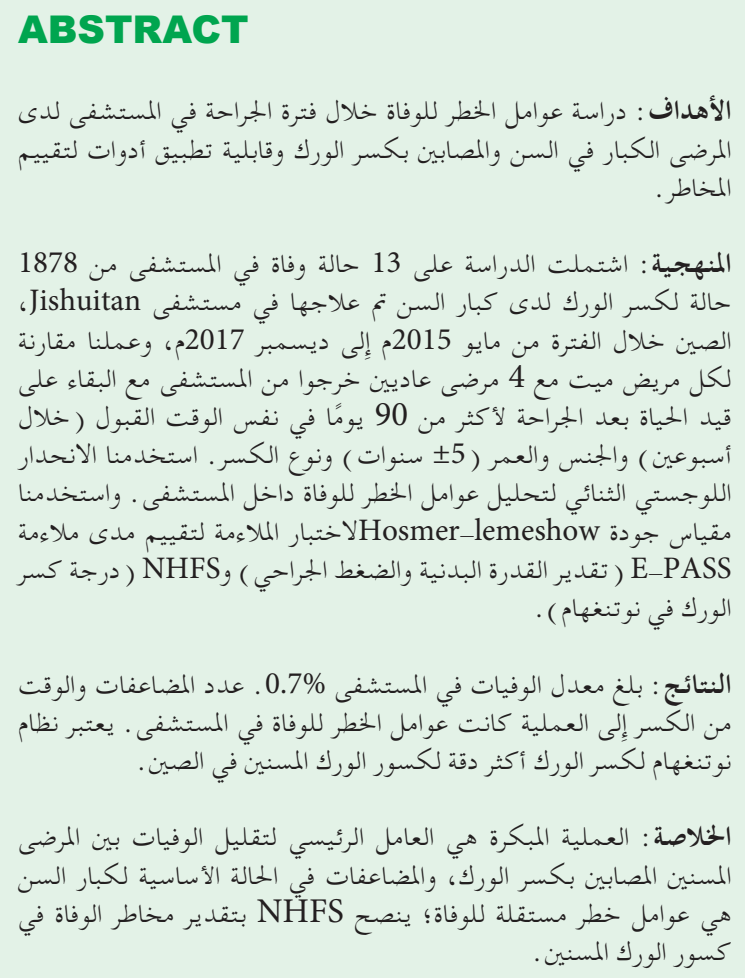

Objectives: To investigate the risk factors of perioperative in-hospital death in elderly patients with hip fracture and the applicability of risk assessment tools.

Methods: Thirteen in-hospital death cases from 1878 geriatric hip fracture treated in Jishuitan Hospital, China from May 2015 to December 2017 were collected, each dead patient was compared with 4 normal discharged patients with a postoperative survival of more than 90 days at the same admission time (within 2 weeks), gender, age $( \pm 5$ years), and fracture type. Binary logistic regression was used to analyze the risk factors of in-hospital death; Hosmerlemeshow goodness of fit test was used to evaluate the goodness of fit of E-PASS (estimation of physical ability and surgical stress) and NHFS (Nottingham hip fracture score).

Results: Mortality in hospital was $0.7 \%$; the number of comorbidities and the time from fracture to operation were the risk factors of in-hospital death. Nottingham hip fracture score system is more accurate to elderly hip fractures in China.

Conclusion: Early operation is the key factor to reduce mortality in elderly patients with hip fracture, and the comorbidities in the basic state of the elderly are the independent risk factors of death; NHFS is recommended to estimate death risk in geriatric hip fractures.

Keywords: hip fractures, in-hospital death, risk factor, risk assessment tools

Saudi Med J 2022; Vol. 43 (2): 197-201 doi: 10.15537/smj.2022.43.2.20210717

From the Department of Geriatrics (Zhang, Li, Yuan, Li, Liu, Fan), and from Department of Orthopaedics and Traumatology (Yang, Wu), Beijing Jishuitan Hospital, Beijing, China.

Received 8th September 2021. Accepted 26th December 2021.

Address correspondence and reprint request to: Dr. Xinbao Wu, Department of Orthopaedics and Traumatology, Beijing Jishuitan Hospital, Beijing, China. E-mail: wuxinbao_jst@126.com

ORCID ID: https://orcid.org/0000-0003-3136-1688

$\mathrm{T}$ he incidence of hip fracture in China has been increasing significantly in recent years. Studies have shown that from 1990 to 1992 , the incidence rate of hip fracture over 50 years of age was 83/100,000 for male and 80/100,000 for female; from 2002 to 2006, the incidence increased to $129 / 100,000$ for male and 229/100,000 for female. ${ }^{1}$ A new data shows the number 
of hip fractures per 100,000 people age $65+$ was 278 between 2013 and 2016 in China. ${ }^{2}$

Hip fracture is one of the most fatal fractures among elderly fragility fractures. An in-hospital mortality of $2.71 \%$ in elderly hip fractures from 2004 to 2014 has been reported in China. ${ }^{3}$ Clinicians believe that all-cause mortality can be reduced by early surgery. ${ }^{4,5}$ Especially in recent years, with the participation of Orthogeriatrics, the mortality of elderly hip fracture patients has been further reduced. It can also be seen from the repeated correction of Nottingham hip fracture score (NHFS). ${ }^{6,7}$ Therefore, in the current medical environment and medical mode, the changes in the risk factors for in-hospital death of elderly hip fractures, and the effectiveness of the risk assessment tools used in the past are the issues to be discussed in this study.

Methods. When patients admitted to the emergency, a pelvic $\mathrm{x}$-ray is made at first; after hip fracture was clear, a geriatrician began preoperative consultation. All the patients over 65 years will be enrolled in geriatric trauma unit belong to trauma orthopedics, and geriatrics and orthopedics were managed together throughout the hospitalization. No surgery during weekends. Early mobilization after surgery with a physiotherapist was arranged on the first day from postoperative. In the case of stable condition, most patients were discharged the second day after surgery, with the anti osteoporosis treatment regimen. The average length of stay was 4 days.

Death group. Of the 13 patients, 8 were males and 5 were females, with a mean age of $84.2 \pm 3.9$ years $(79-90$ years); there were 6 cases of femoral neck fracture and 7 cases of intertrochanteric fracture.

Survival group. Each dead patient was compared with 4 normal discharged patients with a postoperative survival of more than 90 days at the same admission time (within 2 weeks), gender, age ( \pm 5 years), and fracture type. Therefore in the 52 matched control patients, 32 were males and 20 were females, with a mean age of

Disclosure. This study was funded in 2015 by the Special Fund for Clinical Medicine Development of Beijing Municipal Administration of Hospitals, China (ZYLX201506); 2019 Beijing Health Care Research Project (Jing 19-17); and 2019 Scientific Research Cultivating Plan of Beijing Municipal Administration of Hospitals, China (PX2019015).
83.6 \pm 3.9 years (76-91 years); there were 24 cases of femoral neck fracture and 28 cases of intertrochanteric fracture.

The inclusion criteria of the study were: i) patients aged $\geq 65$ years; ii) patients diagnosed with hip fracture; and iii) patients injured within 21 days from the time of seeing a doctor. While the exclusion criteria were: i) tumor metastasis caused pathological fractures; ii) patients with periprosthetic fracture; and iii) patients who have not been hospitalized.

Estimation of physiologic ability and surgical stress $($ E-PASS $)$ mortality $=13.362$ preoperative risk score (PRS) 2-11.277 (PRS) + 1.969; PRS=-0.0686+0.00345 age +0.323 severe heart disease (yes 1 , no 0 ) +0.205 severe pulmonary disease (yes 1 , no 0$)+0.153$ diabetes mellitus (yes 1 , no 0) +0.148 SI index +0.0666 American Society of Anesthesiologists (ASA) .

Status index (SI) level 0-4. According to the definition standard of the eastern cancer cooperation group, level 0 is fully active and can perform all activities without restrictions; level 1 is restricted physical activity, active, able to perform a light physical or sedentary nature of work (such as light housework and office work); level 2 refers to being able to take care of themselves, but unable to work for $50 \%$ or more of the time when they are awake; level 3 means only limited self-care and sitting or lying ill in bed more than $50 \%$ of the time awake. Level 4 means completely unable to take care of themselves or completely confined to bed or chair.

According to the ASA score, grade 1 is a normal healthy person; grade 2 is a mild systemic disease; grade 3 is a serious systemic disease without disability; grade 4 is a disabling systemic disease, which is life-threatening; and grade 5 is dying, whether the operation or not will not survive for 24 hours.

Nottingham hip fracture score adopts seven parameters before operation: age, gender, admission hemoglobin, mini mental test score (MMTS), place of residence, number of basic comorbidities, and whether it is complicated with malignant tumors. One or 3 points are assigned respectively to calculate the total NHFS score, and then calculate the 30 day death risk through the formula: $=100 /\left[1+\mathrm{e}^{(5.012 \times(\mathrm{NHFS} \times 0.481))}\right]$

Statistical analysis. The perioperative in-hospital death or survival was used as the dependent variable, and the risk factors that might have an impact on the outcome were used as independent variables, including: comorbidity (Charlson comorbidity index score, CCI), history of smoking, history of falls, on crutches or not, type of anesthesia, duration of surgery, amount of bleeding, time from fracture to surgery, body mass 
index, femoral neck bone density T score, ASA score, admission hemoglobin level, and admission albumin level. The SPSS for Windows, version 23 (IBM Corp., Armonk, N.Y., USA) was used for statistical analysis, and the binary logistic regression analysis method (Forward step likelihood ratio) was used to screen the risk factors for death. The test level value was 2-sided $p<0.05$.

The receiver operating characteristic (ROC) curve was plotted, with perioperative in-hospital death or survival as the dependent variable, 2 risk scoring tools, E-PASS and NHFS as independent variables. The area under (AUC) the ROC was used to test the predictive ability of the 2 risk scoring tools, E-PASS and NHFS, and an AUC $>0.75$ indicated that the scoring tool had sufficient discriminative ability. The Hosmer-Lemeshow goodness-of-fit test was used to assess whether the predicted probability calculated with the scoring tool fit the actual probability. $P<0.05$ indicated that significant difference exist between observed and predicted model, and the scoring model worked poorly; $p>0.05$ indicated that the scoring model worked well.

The study is in accordance to principles of Helsinki Declaration, and was approved by the Beijing Jishuitan Hospital Ethical Review Board (approbation number 201907-09-02).

Results. General information of in-hospital deaths and direct causes of death. From May 2015 to December 2017, a total of 1878 patients with elderly hip fractures who were eligible for the inclusion/exclusion criteria were admitted to the geriatric orthopedic department, Beijing Jishuitan Hospital, with a mean age of 79.6 years (65-105 years), there were $13(0.7 \%)$ cases of in-hospital death.

Of the 13 in-hospital deaths, there were 4 cases of death before the surgery, including 3 cases of intertrochanteric fracture and one case of femoral neck fracture; all the patients with femoral neck fracture underwent femoral head replacement. The direct causes of death included: 7 (54\%) cases of lung infection, $2(15 \%)$ cases of acute myocardial infarction, $2(15 \%)$ cases of acute erosive hemorrhagic gastritis, $1(8 \%)$ case of acute respiratory distress syndrome, and $1(8 \%)$ case of suspected acute pulmonary embolism. The general information of the deaths is shown in Table 1.

Binary logistic regression analysis. Multivariate binary logistic regression analysis showed that the number of comorbidities and the time from fracture to surgery were independent risk factors for perioperative death in elderly patients with hip fracture, as shown in Table 2. Of these, the risk of perioperative death increased by 2.7-fold for each additional comorbid chronic disease in the CCI, and 1.4-fold for each additional day from fracture to surgery.

Death risk factor scores. For both E-PASS mortality and NHFS death risk assessment tools, the AUC (under ROC) value was used to judge the predictive effectiveness. The AUC for the E-PASS mortality assessment tool was 0.758 (95\% CI 0.635,0.881); the AUC for the NHFS death risk assessment tool was 0.769 (95\% CI 0.604,0.934); see Figure 1. The HosmerLemeshow goodness-of-fit test was used for validation, with $p=0.182$ for E-PASS mortality assessment tool, and $p=0.335$ for NHFS death risk assessment tool. It shows that both the above scoring models work well, and the NHFS death risk assessment tool is slightly superior.

Table 1 - General information of death group and survival group in elderly hip fractures patients.

\begin{tabular}{lccc}
\hline Variables & Death & Survival & $P$-value \\
\hline BMI $\left(\mathrm{kg} / \mathrm{m}^{2}\right)$ & $21.56 \pm 5.93$ & $22.35 \pm 3.27$ & 0.650 \\
$\begin{array}{l}\text { Days form fracture to } \\
\text { surgery }\end{array}$ & $5.89 \pm 4.70$ & $4.55 \pm 2.98$ & 0.261 \\
Hours of surgery & $77.22 \pm 20.78$ & $87.75 \pm 23.67$ & 0.217 \\
Surgery bleeding (ml) & $238.89 \pm 121.91$ & $219.80 \pm 122.52$ & 0.668 \\
T-value of femoral neck & $-2.30 \pm 3.37$ & $-2.68 \pm 0.93$ & 0.745 \\
CCI & $2.39 \pm 1.61$ & $1.37 \pm 1.14$ & 0.048 \\
Mortality predicted by & $5.26 \pm 2.82$ & $3.28 \pm 3.77$ & 0.082 \\
E-PASS(\%) & & \\
$\begin{array}{l}\text { Mortality predicted by } \\
\text { NHFS(\%) }\end{array}$ & $9.30 \pm 4.02$ & $5.68 \pm 1.86$ & 0.007 \\
\hline $\begin{array}{l}\text { T-value: comparison of bone mass density reference values between } \\
\text { patients and young people of the same gender and race. P-value: T-test } \\
\quad \text { of death group and survival group. BMI: body mass index, CCI: }\end{array}$ \\
$\begin{array}{l}\text { Charlson comorbidity index score, E-PASS: estimation of physiologic } \\
\quad \text { ability and surgical stress; NHFS: Nottingham fracture score }\end{array}$ \\
\hline \multicolumn{4}{c}{}
\end{tabular}

Table 2 - Binary logistic regression analysis of perioperative death factors for elderly hip fractures.

\begin{tabular}{lccccc}
\hline Variables & $\begin{array}{c}\text { Partial } \\
\text { regression } \\
\text { coefficient }\end{array}$ & $\begin{array}{c}\text { Standard } \\
\text { error of } \\
\text { partial } \\
\text { regression } \\
\text { coefficient }\end{array}$ & $\begin{array}{c}\text { Wald } \\
\text { value }\end{array}$ & $P$-value & OR $(95 \% \mathrm{CI})$ \\
\hline CCI & 1.004 & 0.474 & 4.498 & 0.034 & $2.732(1.079,6.897)$ \\
$\begin{array}{l}\text { Days } \\
\text { form } \\
\text { fracture to } \\
\text { surgery }\end{array}$ & 0.308 & 0.144 & 4.553 & 0.033 & $1.361(1.026,1.805)$ \\
\hline
\end{tabular}

CCI: Charlson comorbidity index score; OR: odds ratio; CI: confidence interval 


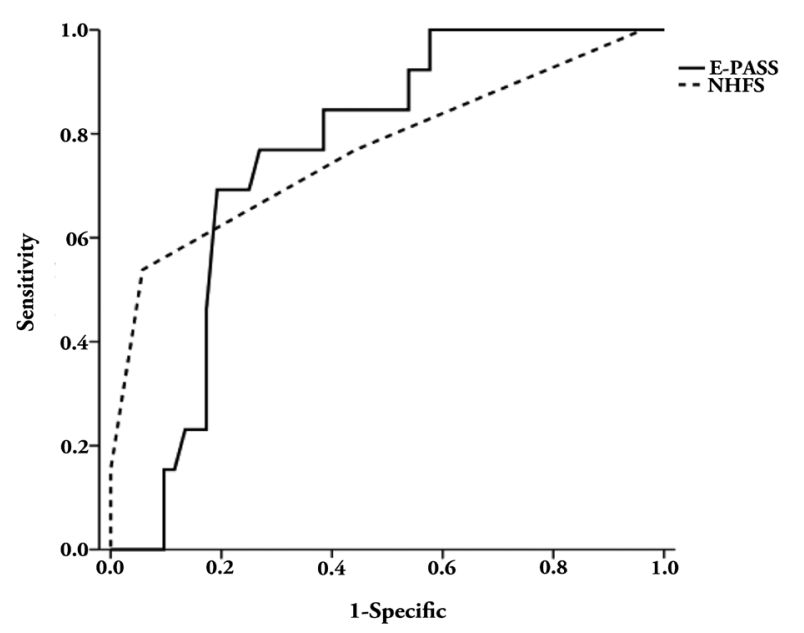

Figure 1 - The receiver operating characteristic curve by E-PASS and NHFS scoring system to assess the risk of death in elderly patients with hip fractures. E-PASS: estimation of physiologic ability and surgical stress, solid line; NHFS: Nottingham fracture score

Discussion. It is currently believed that elderly patients with hip fracture can have better prognosis with the adoption of the orthopedic-geriatric "comanagement model", ${ }^{8}$ and all patients included in this article were admitted under the co-management model, with an in-hospital mortality of $0.69 \%$, which is significantly lower than that in previous reports, ${ }^{9}$ therefore, it is worth popularizing this management model.

A retrospective analysis of attributable mortality included patients from 2009 to 2018, which was relatively recent data. The statistical analysis suggested that baseline characteristics (including age, gender, comorbidity, automaticity, and fracture type), serious postoperative complications, perioperative transfusion, and delayed surgery accounted for $62.4 \%, 11.9 \%$, $9.6 \%$, and $2.7 \%$ of the 6 -month attributable mortality, respectively. ${ }^{10}$ In this study, with age, gender, and fracture type being matched, comorbidity and time from fracture to surgery are risk factors, which is consistent with the result of the study. This also suggests the need to carefully assess comorbidities and inform the patient and patient's families of the risks when receiving elderly patients with hip fracture. In addition, although the improvement of medical knowledge of the public and the popularization of national medical insurance have made it possible for patients to see a doctor in a timely manner, the pain of the elderly is often not notable, or fractures that are likely to have occurred are ignored, resulting in seeing a doctor late and prolonged bedridden time. There is a need to popularize medical knowledge in this aspect so that the public can understand the need for an early imaging test after the elderly fall to exclude activity impairment caused by fractures. According to recent reports in the literature, standardized by the world population composition in 2010, the incidence of hip fractures among people over 65 years in China from 2013 to 2016 is 375 per 100,000 for females, and 203 per 10,000 for males, which has far exceeded the previously reported incidence of hip fractures in China. ${ }^{2}$ Therefore, the task of preventing fragility fractures and reducing perioperative mortality following fractures still has a long way to go.

To overcome the poor predictive accuracy of univariate predictors, some scholars have proposed some scoring models for evaluating the risk of death in hip fractures, and 2 risk scoring systems, E-PASS and NHFS, were used to predict perioperative mortality in this study, respectively. Estimation of physiologic ability and surgical stress is non-specific for hip fractures, which involves preoperative risk score (PRS), surgical stress score and comprehensive risk score; a prediction formula for postoperative mortality was generated based on the PRS, postoperative mortality $=13.362 \mathrm{PRS} 2+$ 11.277PRS + 1.969; the time from surgery for patients observed when the author speculated the formula was $17.3 \pm 12.0(0-142)$ days, thus reflecting to some extent the in-hospital mortality. The evaluation of preoperative physiological conditions regarding E-PASS does not involve neuropsychiatric diseases that have a great impact on the prognosis of elderly hip fractures. At present, it is agreed that the scoring system overestimates the mortality in the prediction for low-risk patients, and a relatively accurate mortality is shown in the prediction for high-risk patients. Nottingham hip fracture score, deriving from the analysis and summary based on a population of hip fractures, is the most commonly used score to predict the perioperative risk for hip fractures; the NHFS uses 7 preoperative parameters: age, gender, admission hemoglobin, admission MMTS, place of residence before admission, number of underlying comorbidities, concomitant malignancy or not, each of which is assigned a score of 1 or 4 to calculate the total NHFS score, and then the risk of death within 30 days is calculated by the formula: $=100$ / $[1+\mathrm{e}(5.012 \times(\mathrm{NHFS} \times 0.481))]$. This value has been verified in one year after surgery ${ }^{11}$ and in the early discharge period; ${ }^{12}$ NHFS is superior to other scoring systems in calculating perioperative comorbidities and mortality, ${ }^{13}$ giving the mortality risk prediction value at 30 days for a single patient; but it still cannot 
play a decisive role in the clinical decision-making for individual patients. Generally, when the predicted mortality exceeds $10 \%$, patients and their families are advised to choose conservative treatments.

The 13 patients in this study were retrospectively assessed using both the E-PASS and NHFS scoring systems, which also indicated that NHFS was more accurate in the assessment of mortality. The NHFS score was also based on the patient's 7 underlying conditions on admission, of which a higher score was assigned with an older age, such as: age $\geq 86$ years was assigned a score of 4 , while CCI $\geq 2$ was assigned a score of 1 . Under the current medical level, whether the age still needs to maintain such a high weight remains to be discussed in the context of big data.

The small number of death cases and the retrospective risk score are the limits of this paper. Subsequent long-term follow-up and prospective studies can provide better data.

In conclusion, with the same age, gender, and fracture type being matched for dead elderly patients with hip fractures, a logistic regression analysis is performed, indicating that the comorbidity and the time from fracture to operation are risk factors for death under the co-management of orthopedics and geriatrics. Two relatively simple surgical risk assessment tools are used to conduct a retrospective evaluation. The results suggest that NHFS is relatively suitable for Chinese elderly population, but it is proposed that the surgical risk assessment formula for the Chinese population should be generated based on big data so as to perform better perioperative diagnosis and treatment to reduce the mortality of elderly hip fractures.

Acknowledgment. We would like to thank Beijing Yiyutang Consulting Service Co., Ltd (http://www.easytowntrans.com) for English language editing.

\section{References}

1. Chinese Medical Association. Guidelines for the diagnosis and treatment of primary osteoporosis (2017). Chin J osteoporosis \& bone miner res 2017: 20: 413-443.
2. Gong XF, Li XP, Zhang LX, Center JR, Bliuc D, Shi Y, et al. Current status and distribution of hip fractures among older adults in China. Osteoporos Int 2021; 32:1785-1793.

3. Chang X, Zhang Bzh, Weng Xsh et al. Risk factor analysis for postoperative mortality in hospital of geriatric hip fracture patients. Chin J Geriatr Orthop Rehabil (Electronic Edition) 2017; 3: 43-49.

4. Abtahi S, Burden AM, Geusens P, et al. The association of oral bisphosphonate use with mortality risk following a major osteoporotic fracture in the United Kingdom: population-based cohort study. J Am Med Dir Assoc 2020; 21:811-816.

5. Li W, Yang M. Fragility Fracture Network (FFN)-China successfully held forum to support FFN Global Call to Action to improve the care of people with fragility fractures. Aging Med (Milton) 2018; 1: 280-281.

6. Maxwell MJ, Moran CG, Moppett IK. Development and validation of a preoperative scoring system to predict 30 day mortality in patients undergoing hip fracture surgery. $\mathrm{Br} \mathrm{J}$ Anaesth 2008; 101: 511-517.

7. Marufu TC, White SM, Griffiths R, Moonesinghe SR, Moppett IK. Prediction of 30-day mortality after hip fracture surgery by the Nottingham hip fracture score and the surgical outcome risk tool. Anaesthesia 2016; 71: 515-521.

8. Civinini R, Paoli T, Cianferotti L, Cartei A, Boccaccini A, Peris A, et al. Functional outcomes and mortality in geriatric and fragility hip fractures-results of an integrated, multidisciplinary model experienced by the "Florence hip fracture unit". Int Orthop 2019; 43: 187-192.

9. Sheehan KJ, Sobolev B, Guy P, Kuramoto L, Morin, Jason M. Sutherland SN, et al. In-hospital mortality after hip fracture by treatment setting. CMAJ 2016; 188: 1219-1225.

10. Zerah L, Hajage D, Raux M, Cohen-Bittan J, Mézière A, Khiami F, et al. Attributable mortality of hip fracture in older patients: A retrospective observational study. J Clin Med 2020; 9: 2370 .

11. Wiles MD, Moran CG, Sahota O, Moppett IK. Nottingham Hip Fracture Score as a predictor of one year mortality in patients undergoing surgical repair of fractured neck of femur. Br J Anaesth 2011; 106: 501-504.

12. Moppett IK, Wiles MD, Moran CG, Sahota O. The Nottingham hip fracture score as a predictor of early discharge following fractured neck of femur. Age Ageing 2012; 41: 322-326.

13. Marufu TC, Mannings A, Moppett IK. Risk scoring models for predicting peri-operative morbidity and mortality in people with fragility hip fractures: Qualitative systematic review. Injury 2015; 46: 2325-2334. 